\title{
Muscular (hypertrophic) subaortic stenosis (hypertrophic obstructive cardiomyopathy): the evidence for true obstruction to left ventricular outflow
}

\author{
E. Douglas Wigle, Mark Henderson, Harry Rakowski and Susan Wilansky \\ Division of Cardiology, Department of Medicine, Toronto General Hospital, and University of Toronto, Toronto, \\ Ontario, Canada
}

\begin{abstract}
Summary: The clinical and haemodynamic significance of the subaortic pressure gradient in patients with muscular (hypertrophic) subaortic stenosis (hypertrophic obstructive cardiomyopathy) has long been debated. In this report we summarize the evidence which indicates that true obstruction to left ventricular outflow exists in these patients.

Rapid left ventricular ejection, through an outflow tract narrowed by ventricular septal hypertrophy, results in Venturi forces causing systolic anterior motion of the anterior (or posterior) mitral leaflets. Mitral leaflet-septal contact results in obstruction to outflow and the accompanying mitral regurgitation. The time of onset of mitral leaflet-septal contact determines the magnitude of the pressure gradient and the severity of the mitral regurgitation, as well as the degree of prolongation of left ventricular ejection time and the percentage of left ventricular stroke volume that is ejected in the presence of an obstructive pressure gradient. Early and prolonged mitral leaflet-septal contact results in a large pressure gradient, significant mitral regurgitation, as well as dramatic prolongation of the ejection time and a large percentage of left ventricular stroke volume being obstructed. Late and short mitral leaflet-septal contact results in little haemodynamic perturbation. Hypertrophic cardiomyopathy patients with obstructive pressure gradients are significantly more symptomatic than those without. Thus the obstructive pressure gradients in hypertrophic cardiomyopathy are of clinical as well as haemodynamic significance. To deny the existence of obstruction to outflow in patients with muscular subaortic stenosis is to deny these patients appropriate medical and surgical therapy.
\end{abstract}

For approximately 20 of the 27 years that hypertrophic cardiomyopathy has been recognized as a clinical entity, there has been active, and at times heated, debate as to the clinical and haemodynamic significance of the outflow tract pressure gradient encountered in patients with muscular subaortic stenosis. We have recently completed extensive reviews of this subject which are summarized in this report (Wigle et al., 1985a,b). From the information presently available, we continue to believe that there is overwhelming evidence in support of true obstruction to left ventricular outflow in hypertrophic cardiomyopathy patients with obstructive subaortic pressure gradients. This subaortic obstruction is important in its own right with regard to causing abnormalities of systolic function in muscular subaortic stenosis, but becomes even more important when it is realized that

Correspondence: E.D. Wigle, M.D., Director, Division of Cardiology, 12-217 Eaton North, Toronto General Hospital, 200 Elizabeth St., Toronto, Ontario, Canada M5G 2C4 the obstruction to outflow also contributes to impaired relaxation, which, along with increased chamber stiffness (decreased compliance), causes the important abnormalities of diastolic function that are so characteristic of hypertrophic cardiomyopathy (Wigle et al., 1985a,b).

Nature of intraventricular pressure differences in man

There are essentially four types of intraventricular pressure differences that may be encountered in hypertrophic cardiomyopathy (Wigle et al., 1985a,b). The impulse gradient due to early systolic ejection (Murgo et al., 1980) and the pressure gradient due to midventricular obstruction (at the level of the papillary muscles) can easily be distinguished from the outflow tract pressure gradient encountered in muscular subaortic stenosis (Wigle et al., 1985a,b). Of greater importance is to distinguish between an intraventricular pressure difference due to cavity obliteration from the obstructive pressure gradient 
seen in muscular subaortic stenosis. This can readily be done by means of transseptal left heart catheterization and utilization of the left ventricular inflow tract pressure concept (Wigle et al., 1967a) (Figure 1). In addition to the inflow tract pressure concept there are many ancillary methods that permit distinction between these two types of intraventricular pressure difference (Wigle et al., 1967a, 1985a,b). In all of our studies of the haemodynamics of muscular subaortic stenosis we have used the inflow tract pressure concept, and ancillary measures, to ensure that we are dealing with an obstructive pressure gradient.

\section{Mechanism of the obstructive subaortic pressure gradient in muscular subaortic stenosis}

Figure 2 illustrates our current concept with regard to the mechanism of the obstruction to left ventricular

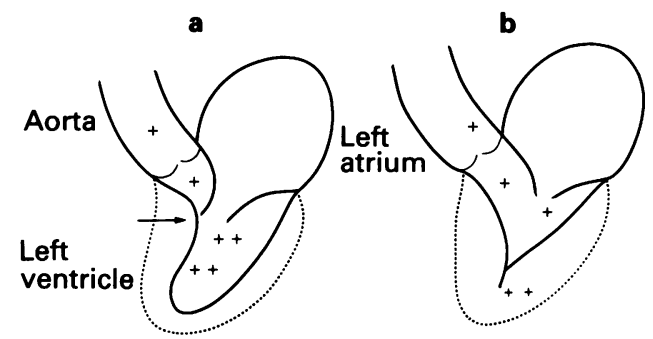

Figure 1 The left ventricular inflow tract pressure concept. (a) In muscular subaortic stenosis, because the obstruction to left ventricular outflow (arrow) is caused by anterior mitral leaflet-ventricular septal apposition, the intraventricular pressure distal to the stenosis (and proximal to the aortic valve) is low $(+)$ whereas all ventricular pressures proximal to the stenosis, including the one just inside the mitral valve (the inflow tract pressure) are elevated $(++)$. (b) When an intraventricular pressure difference is recorded, due to catheter entrapment by the myocardium as the result of cavity obliteration, the elevated ventricular pressure is recorded only in the area of cavity obliteration $(t+)$. The intraventricular systolic pressure in all other areas of the left ventricular cavity, including that in the inflow tract, just inside the mitral valve, is low $(+)$ and equal to the aortic systolic pressure. Note that in cavity obliteration, there is an intraventricular pressure difference between the apex and the inflow tract and also the outflow tract. In muscular subaortic stenosis there is no intraventricular pressure difference between the apex and the inflow tract as they are both elevated above the outflow tract pressure. The three areas of the left ventricle represented by the $+s$ in each of these diagrams are, from above downward, the outflow tract just below the aortic valve (subaortic region), the inflow tract just inside the mitral valve, and the left ventricular apex (see text). (Reprinted with permission from Wigle et al. 1985, Progress in Cardiovascular Diseases, 28, 1.) outflow in muscular subaortic stenosis. In essence, the hypertrophy of the upper ventricular septum narrows the left ventricular outflow tract causing the ejection of blood from the left ventricle to occur at a high velocity and the ejection path to be closer to the mitral leaflets than is normal. As a result of this, the anterior (or posterior) mitral leaflet is drawn into the outflow tract by a Venturi effect (Wigle et al., 1970). Mitral leafletseptal contact results in obstruction to left ventricular outflow and the mitral regurgitation is caused by the systolic anterior motion of the mitral leaflet.

The evidence supporting the concept that mitral leaflet systolic anterior motion is caused by a Venturi effect and that mitral leaflet-septal contact results in obstruction to left ventricular outflow and mitral regurgitation may be summarized as follows (Wigle et al., 1985a,b): (1) Hypertrophic cardiomyopathy patients with obstructive pressure gradients have been demonstrated to have thicker ventricular septa and narrower outflow tracts at the onset of systole than do patients without pressure gradients. (2) Patients with pressure gradients demonstrate early and prolonged mitral leaflet-septal contact (severe systolic anterior motion of the mitral leaflets) whereas patients without pressure gradients do not. (3) The onset of the obstructive pressure gradient (defined as the peak of
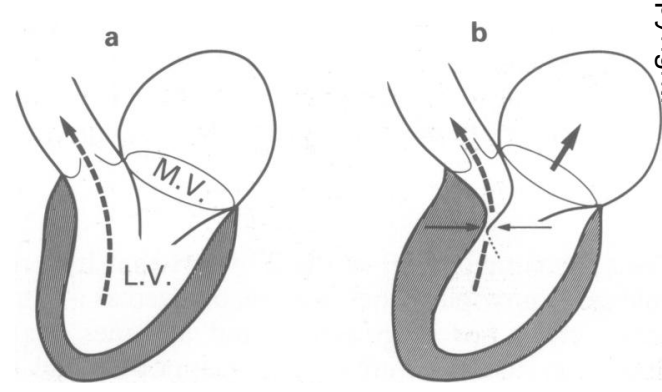

Figure 2 Proposed mechanism of systolic anterior motion of the anterior mitral leaflet in muscular subaortic stenosis. In normals (a) blood is ejected from the left ventricle in a relatively direct path into the aorta, through a wide open outflow tract. In muscular subaortic stenosis, (b) the ventricular septum is thickened (left horizontal arrow) resulting in a narrowed outflow tract. Because of this narrowing, the ejection of blood from the ventricle occurs at a high velocity and the ejection path is closer to the anterior mitral leaflet than is normal. As a result of this, the anterior leaflet is drawn into the outflow tract towards the septum, by a Venturi effect (right horizontal arrow). Mitral leaflet-septal contact results in obstruction to left ventricular outflow. Mitral regurgitation (upper right oblique arrow) results from the anterior mitral leaflet being out of its normal systolic position. $\mathrm{LV}=$ left ventricle, $M V=$ mitral valve. (Reprinted with permission from Wigle et al., 1985, Progress in Cardiovascular Diseases, 28, 1. 
the aortic percussion wave) commences virtually simultaneously with the onset of mitral leaflet-septal contact (Figure 3). The magnitude of the pressure gradient is related to the time of onset of mitral leafletseptal contact and to its duration. Thus, large pressure gradients are associated with early and prolonged mitral leaflet-septal contact (Figure 3) whereas small pressure gradients are associated with late onset and brief mitral leaflet-septal contact. (4) Mitral regurgitation is present in all patients with obstructive pressure gradients. The mitral leak commences with the development of mitral leaflet systolic anterior motion and is greatest during the period of mitral leaflet-septal contact. The degree of mitral regurgitation and the magnitude of the pressure gradient both vary directly with the severity of mitral leaflet systolic anterior motion. Hypertrophic cardiomyopathy patients without pressure gradients (and no mitral leaflet systolic anterior motion) tend to have either trivial or no mitral regurgitation. (5) Mitral leaflet systolic anterior mo-

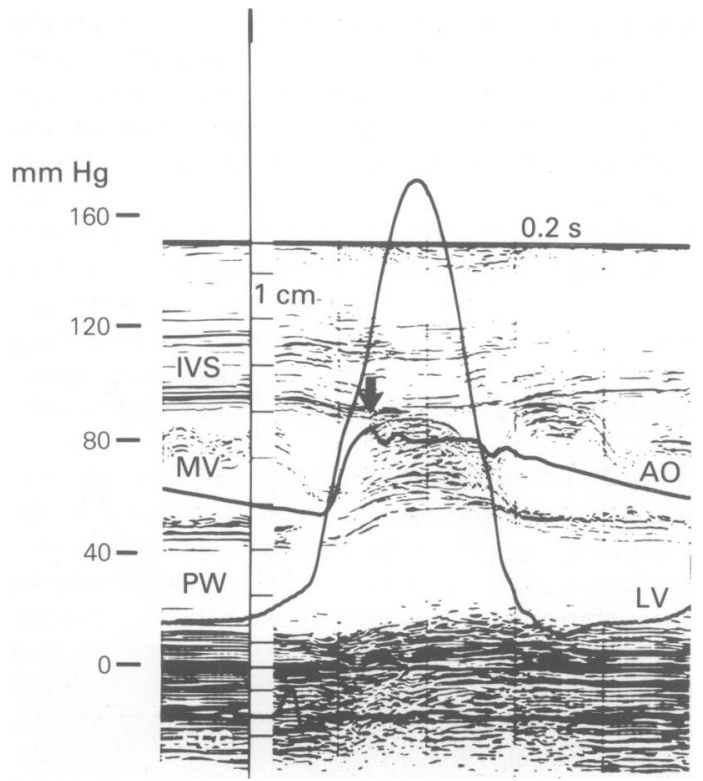

Figure 3 Simultaneous haemodynamic and one-dimensional echocardiographic recordings in a patient with muscular subaortic stenosis (gradient $86 \mathrm{~mm} \mathrm{Hg}$ ). The arrow indicates the onset of mitral leaflet-septal contact and the onset of the pressure gradient (defined as the peak of aortic percussion wave), which are virtually simultaneous. Note how early in systole the onset of mitral leafletseptal contact and the pressure gradient occur in patients with severe outflow tract obstruction. IVS = interventricular septum; $\mathbf{M V}=$ mitral valve; $\mathrm{PW}=$ posterior wall; $A O=$ central aortic pressure; $L V=$ left ventricular pressure. (From Pollick et al. 1982, Circulation, 66, 1087. With permission of the American Heart Association, Inc.) tion has been demonstrated in an experimental model of the left ventricle in muscular subaortic stenosis when ejection was sufficiently rapid and the path of ejection close enough to the mitral leaflets (Bellhouse \& Bellhouse, 1972). (6) Interventions which increase the velocity of ejection (increased contractility, decreased afterload) increase the severity of mitral leaflet-septal contact as well as increasing the magnitude of the pressure gradient and the mitral regurgitation. Interventions which decrease the velocity of ejection (decreased contractility, increased afterload) decrease the severity of mitral leaflet-septal contact with concomitant lessening of the pressure gradient and mitral regurgitation. Interventions which increase outflow tract size (blood volume expansion, the ventriculomyotomy-myectomy operation) reduce or abolish the mitral systolic anterior motion and hence the pressure gradient and mitral regurgitation. A laterally placed myectomy incision abolishes the systolic anterior motion of the lateral half of the anterior mitral leaflet (where the outflow tract is enlarged) but severe systolic anterior motion remains in the medial half of the leaflet, where the outflow tract is still narrow.

All of these observations are compatible with a Venturi effect being the cause of mitral leaflet systolic anterior motion and with mitral leaflet-septal contact being the cause of the obstructive pressure gradient and the concomitant mitral regurgitation. Other authors have suggested that tethering of the mitral leaflets, posterior wall hyperkinesis or cavity obliteration (Criley \& Seigel, 1985) may cause systolic anterior motion of the mitral leaflets. If any of these contraction mechanisms were responsible for mitral leaflet systolic anterior motion then the systolic anterior motion should last until contraction ceases, i.e. until end-systole. We have clearly demonstrated that mitral leaflet-septal contact ends before end-systole. In addition the rate of development of systolic anterior motion is three times the rate of inward movement of the posterior wall, and maximal systolic anterior motion (mitral leaflet-septal contact) occurs several hundred milliseconds before maximal inward movement of the posterior wall. All of these considerations would render it unlikely, if not untenable, that systolic tethering of the mitral leaflets, posterior wall hyperkinesis or cavity obliteration could cause systolic anterior motion of the mitral leaflets.

\section{Significance of the obstructive pressure gradient in muscular subaortic stenosis}

One of the essential features of any form of obstruction to left ventricular outflow must be the fact that a significant proportion of left ventricular emptying occurs in the presence of a pressure gradient, i.e. in the 
presence of obstruction to ejection. Such obstructed ejection represents a systolic overload to the left ventricle. Much of the controversy as to the significance of the subaortic pressure gradients in hypertrophic cardiomyopathy revolves around whether a significant portion of left ventricular emptying occurs in the presence of a pressure gradient. Everyone acknowledges that there is early rapid non-obstructed ejection in muscular subaortic stenosis (Figure 2). The question is what percentage of left ventricular ejection occurs after the onset of mitral leaflet-septal contact and the pressure gradient. Detailed studies using cineangiographic (Wigle et al., 1985a,b), echocardiographic (Glasgow et al., 1980), combined nuclear angiographic and micromanometric (Bonow et al., 1984), as well as electromagnetic (Ross et al., 1966) and Doppler (Maron et al., 1985) aortic flow techniques have demonstrated that 40 to $80 \%$ of left ventricular stroke volume is ejected in the presence of a pressure gradient in muscular subaortic stenosis. We have demonstrated that the time of onset of mitral leaflet-septal contact not only determines the magnitude of the pressure gradient but also the percentage of stroke volume that is ejected against the obstruction and the degree of prolongation of left ventricular ejection time (Wigle et al., 1985a,b). Thus, when mitral leaflet-septal contact occurs early in systole there is a large pressure gradient, and a large proportion of left ventricular stroke volume is ejected in the presence of obstruction and the left ventricular ejection time is greatly prolonged. When mitral leaflet-septal contact occurs later in systole the pressure gradient is smaller, the percentage of stroke volume that is obstructed is dramatically less and left ventricular ejection time is only mildly prolonged. There is no obstruction to left ventricular outflow if mitral leaflet-septal contact occurs after 55 percent of the left ventricular ejection time (systolic ejection period). The fact that a significant percentage of left ventricular ejection occurs in the presence of a pressure gradient in muscular subaortic stenosis is crucial to the understanding of the haemodynamic significance of these pressure gradients in hypertrophic cardiomyopathy. It must also be appreciated that the left ventricle is emptying into the left atrium (mitral regurgitation) as well as into the aorta in the presence of a pressure gradient. Indeed cineangiographic observations indicate that the mitral regurgitation occurs principally in the last half of systole and contributes significantly to the small end-systolic volume that is often seen in patients with muscular subaortic stenosis (Wigle et al., 1985a,b).

A very important characteristic of obstruction to left ventricular outflow is a prolongation of left ventricular ejection time. Knowing that a significant proportion of left ventricular emptying occurs in the presence of pressure gradient and hence obstruction to outflow, it is not surprising that left ventricular ejection time in prolonged in muscular subaortic stenosis in direct relation to the magnitude of the pressure gradient (Wigle et al., 1967b). Indeed, the time of onset of mitral leaflet-septal contact determines both the magnitude of the pressure gradient and the degree of prolongation of left ventricular ejection time, as well as the percentage of stroke volume that is obstructed (vide supra). Interventions which decrease the pressure gradient (increased afterload, decreased contractility, successful surgery) also decrease the left ventricular ejection time, as would be expected with relief of obstruction to outflow. Interventions which increase the pressure gradient (decreased afterload, increased contractility) result in prolongation of the left ventricular ejection time, as would be expected with increased obstruction to outflow. The prolongation of ejection time in hypertrophic cardiomyopathy patients with obstructive pressure gradients is even more impressive when it is recalled that all patients with muscular subaortic stenosis have mitral regurgitation which, in and of itself tends to shorten the ejection time. It is also important to note that in cavity obliteration there is an inverse relationship between the magnitude of the intraventricular pressure difference and the left ventricular ejection time, whereas in muscular subaortic stenosis there is a direct relationship between the magnitude of the pressure gradient and the degree of prolongation of the ejection time (Wigle et al., 1967b). The prolongation of ejection time in muscular subaortic stenosis has now been demonstrated by clinical, phonocardiographic, echocardiographic, haemodynamic and Doppler techniques.

If obstruction to left ventricular outflow in muscular subaortic stenosis is a significant factor in the pathophysiology of hypertrophic cardiomyopathy, then patients with obstruction to outflow should be symptomatically worse than those without obstruction to outflow. Some authors have suggested that there is no difference in symptomatology between those with and without obstructive pressure gradients (Criley \& Siegel, 1985; Murgo et al., 1980). Recently, however, we have analysed the clinical features of hypertrophic cardiomyopathy patients with and without obstructive pressure gradients and have found that patients with obstruction have a significantly higher incidence of dyspnoea, angina, presyncope-syncope, as well as more disabling symptoms, than do patients without obstruction to outflow (Wigle et al., 1985a,b). In addition, hypertrophic cardiomyopathy patients with muscular subaortic stenosis have a significantly greater incidence of Grade 3-4/6 apical systolic murmurs and reversed splitting of the second heart sound than do patients with non-obstructive hypertrophic cardiomyopathy. These observations indicate that obstruction to left ventricular outflow is of 
clinical as well as haemodynamic significance in hypertrophic cardiomyopathy and is in keeping with the dramatic relief of symptoms that follows successful ventriculomyotomy-myectomy surgery.

\section{The non-obstructive viewpoint}

Criley \& Siegel (1985), Goodwin (1982), and Murgo et al. (1980) have been prominent in espousing the nonobstructive viewpoint in hypertrophic cardiomyopathy, but for different reasons. Criley \& Siegel (1985) believe that the intraventricular pressure differences in hypertrophic cardiomyopathy are related to cavity obliteration and not to obstruction to outflow, or to entrapment of the catheter recording the elevated ventricular pressure in an area of cavity obliteration. They suggest that a pressure difference is created between rapidly and slowly emptying regions of the ventricle but do not provide a scientific basis for this claim. They have suggested that mitral leaflet systolic anterior motion and aortic valve notching are caused by posterior wall hyperkinesis or cavity obliteration, but these suggestions are untenable at present (vide supra). It would be our belief that the intraventricular pressure differences in Criley's studies are either due to catheter entrapment in obliterated areas of the myocardium or are true obstructive pressure gradients and we have described methods to distinguish between these two types of intraventricular pressure difference (Figure 1) (Wigle et al., 1985a,b). Of importance is the fact that many cases of muscular subaortic stenosis do not demonstrate abnormally high ejection fractions. In such cases it would be rather difficult to ascribe the pressure gradient to cavity obliteration when in fact there is none! It is important to realize that cavity obliteration is not a disease entity, but rather a non-specific manifestation of left ventricular hypertrophy and is often, but not invariably, present in hypertrophic cardiomyopathy. The degree of cavity obliteration in any form of left ventricular hypertrophy is related to the degree of hypertrophy, left ventricular systolic function, and to the degree of obstruction to left ventricular outflow and the presence or absence of mitral regurgitation. Goodwin (1982) speaks of cavity elimination rather than cavity obliteration, but we would regard his viewpoint as being rather similar to that of Criley \& Siegel (1985).

Murgo et al. (1980) do not believe that there is a haemodynamically significant obstruction to left ventricular outflow in muscular subaortic stenosis in that the aortic velocity flow profiles are similar in hypertrophic cardiomyopathy patients with and without pressure gradients. Recent re-analysis of this work, however, indicates that the onset of the outflow tract obstruction during systole in muscular subaortic stenosis does in fact significantly alter the aortic ejection flow profile in that both the flow time and ejection time become prolonged, when compared to patients without obstruction to outflow. For detailed analysis of both the obstructive and non-obstructive viewpoints the reader is referred to two recent reviews (Wigle et aal., 1985a,b).

\section{Conclusion}

As a result of the foregoing analysis of the mechanism and significance of the obstructive subaortic pressure gradient in muscular subaortic stenosis (hypertrophic obstructive cardiomyopathy) one must conclude that these gradients do in fact indicate an obstruction to left ventricular outflow that is of haemodynamic as well as clinical significance. To deny that there is obstruction to left ventricular outflow in muscular subaortic stenosis is to deny these patients appropriate medical and surgical therapy.

\section{Acknowledgements}

This work was supported in part by the Heart and Stroke Foundation of Ontario. The authors wish to acknowledge the kind secretarial assistance of Miss Sharon Tribble.

\section{References}

BELlHouse, B.J. \& BELLHOUSE, F.H. (1972). The fluid mechanics of subaortic stenosis in a model left ventricle. University of Oxford, Department of Engineering Science, Report No. 1032/72.

BONOW, R.O., OSTROW, D.R., ROSING, D.R., CANNON, R.O., LEON, M.B., WATSON, R.M., BACHARACH, S.L., GREEN, M.V. \& EPSTEIN, S.E. (1984). Dynamic pressure-volume alterations during left ventricular ejection in hypertrophic cardiomyopathy: Evidence for true obstruction to left ventricular outflow. Circulation, 70: II-17 (abstract).

CRILEY, J.M. \& SIEGEL, R.J. (1985). A non-obstructive view of hypertrophic cardiomyopathy. In Heart Muscle Dis- ease, Goodwin, J.F. (ed). p. 157. MTP Press: Lancaster. GLASGOW, G.A., GARDIN, J.M., BURNS, C.S., CHILDS, W.J. \& HENRY, W.L. (1980). Echocardiographic and Doppler flow observations in idiopathic hypertrophic subaortic stenosis (IHSS). Circulation, 62, III-99 (abstract).

GOODWIN, J.F. (1982). The frontiers of cardiomyopathy. British Heart Journal, 48, 1.

MARON, B.J., GOTTDIENER, J.S., ARCE, J., ROSING, D.R., WESLEY, Y.E. \& EPSTEIN, S.E. (1985). Dynamic subaortic obstruction in hypertrophic cardiomyopathy: analysis by pulsed Doppler echocardiography. Journal of the American College of Cardiology, 6, 1. 
MURGO, J.P., ALTER, B.R., DORETHY, J.F., ALTOBELLI, S.A. \& McGRANAHAN, G.M. (1980). Dynamics of left ventricular ejection in obstructive and nonobstructive hypertrophic cardiomyopathy. Journal of Clinical Investigation, 66, 1369.

ROSS, J., BRAUNWALD, E., GAULT, J.H., MASO, D.T. \& MORROW, A.G. (1966). Mechanism of the intraventricular pressure gradient in idiopathic hypertrophic subaortic stenosis. Circulation, 34, 558.

WIGLE, E.D., MARQUIS, Y. \& AUGER, P. (1967a). Muscular subaortic stenosis: initial left ventricular inflow tract pressure in the assessment of intraventricular pressure differences in man. Circulation, 35, 1100.

WIGLE, E.D., AUGER, P. \& MARQUIS, Y. (1967b). Muscular subaortic stenosis: the direct relation between the intraventricular pressure difference and left ventricular ejection time. Circulation, 36, 36.

WIGLE, E.D., ADELMAN, A.G. \& SILVER, M.D. (1970). Pathophysiological considerations in muscular subaortic stenosis. In Hypertrophic Obstructive Cardiomyopathy, Ciba Foundation Study Group 37, Wolstenholme, G.E.W. and O'Connor, M. (eds). p. 63. Churchill: London.

WIGLE, E.D., SASSON, Z., HENDERSON, M.A., RUDDY, T.D. FULOP, J., RAKOWSKI, H. \& WILLIAMS, W.G. (1985a). Hypertrophic cardiomyopathy. The importance of the site and the extent of hypertrophy. A review. Progress in Cardiovascular Diseases, Vol. XXVIII, 1.

WIGLE, E.D., HENDERSON, M.A., SASSON, Z., POLLICK, C. \& RAKOWSKI, H. (1985b). Muscular subaortic stenosis (hypertrophic obstructive cardiomyopathy): the evidence for obstruction to left ventricular outflow. In Heart Muscle Disease, Goodwin, J.F. (ed). p. 217. MTP Press: Lancaster. 